\title{
Effectiveness of a hospital-based postnatal parent education intervention about pain management during infant vaccination: a randomized controlled trial
}

\author{
Anna Taddio BScPhm PhD, Vibhuti Shah MD, Lucie Bucci MA, Noni E. MacDonald MD, \\ Horace Wong MSc, Derek Stephens MSc
}

Cite as: CMAJ 2018 October 22;190:E1245-52. doi: 10.1503/cmaj.180175

\begin{abstract}
BACKGROUND: Parents have reported that they want to learn how to reduce pain in infants during vaccinations. Our objective was to compare different levels of intensity of postnatal education about pain mitigation on parental selfreported use of interventions at future infant vaccinations.
\end{abstract}

METHODS: We conducted a longitudinal, 3-group parallel, add-on, randomized controlled trial on the postnatal ward of a hospital. New mothers, unaware of the hypothesis, were randomly assigned to 1 of 3 intervention groups and 3 follow-up groups (i.e., 9 groups, $3 \times 3$ ). The 3 intervention groups were control (general immunization information), pain pam- phlet (pain mitigation information), and pain pamphlet and pain video (pain mitigation information). Both pain mitigation education groups also received general immunization information. The 3 follow-up groups were 2-, 4- and 6 -month infant vaccinations. Mothers reported use of breastfeeding, sucrose and topical anesthetics during infant vaccinations in a telephone survey.

RESULTS: Of 3420 participants, followup was available for 2549 (75\%): 36.1\%, $34.2 \%$ and $29.7 \%$ reported on pain mitigation practices at 2-, 4- and 6-month vaccinations, respectively $(p=0.9)$. Maternal characteristics did not differ $(p>0.05)$ : mean age, 33.6 years; 58\% were primipara. Utilization of any intervention (breastfeeding, sucrose or topical anesthetics) was 53.2\%, 61.4\% and $63.0 \%$ for control, pain pamphlet, and pain pamphlet and pain video groups, respectively $(p<0.001)$; both pain education groups had higher utilization than the control group, but did not differ from one another. Uptake differed among intervention groups at 2 and 4 months but not at 6 months.

INTERPRETATION: Hospital-based postnatal education increased parental use of pain interventions at infant vaccinations and can be added to existing education. Trial registration: ClinicalTrials. gov, no. NCT01937143.
V accine injections are the most common recurring painful procedure in infancy. Pain experienced by infants during vaccinations can cause distress in infants, caregivers and health providers, contributing to a negative vaccination experience and vaccine hesitancy. ${ }^{1-3} \mathrm{~A}$ clinical practice guideline identified breastfeeding, sweettasting solutions and topical anesthetics as highly effective interventions; ${ }^{4,5}$ however, infants have not benefited broadly from their use. ${ }^{6-8}$ Parents report that they are largely unaware of how to reduce vaccination pain and that this is not routinely discussed with health providers, despite wanting to make their infants more comfortable during vaccination. ${ }^{6}$
Directly targeting education to parents about pain mitigation during infant vaccinations supports family-centred care and has the potential to improve pain care in infants. Reduced pain in infants can, in turn, improve the vaccination experience, promote more positive attitudes about vaccination and reduce vaccine hesitancy. ${ }^{9}$ Parental soothing of infants in distress is also a key feature of responsive caregiving and secure parent-child attachment. ${ }^{10,11}$ Thus, promoting pain mitigation in the vaccination context has the added benefit of reinforcing the critical role of caregivers in regulating infant distress.

With parent input, we developed knowledge translation tools to inform parents about how to reduce infant pain during vaccination, including a pamphlet and video..$^{12,13}$ We implemented the 
pamphlet and video together in prenatal classes and outpatient clinics, and documented increased parental use of pain mitigation interventions at future infant vaccinations. ${ }^{14,15}$ However, the findings of these previous studies have limited generalizability owing to impractical implementation, including delivery methods, and selection bias. Separately, we showed minimal effects when only the pain pamphlet was inserted into the postnatal discharge package given to all new parents in the hospital after the birth of an infant. ${ }^{16}$ It is not known whether the passive nature of the delivery method for the pain pamphlet, ${ }^{17}$ the lack of inclusion of the companion pain video or the hospital setting can explain the negative result.

Hospital-based education after birth is routinely provided to new parents on a variety of topics about baby care and warrants further study as a setting for education about pain mitigation during infant vaccination. ${ }^{18}$ The hospital offers several important advantages over other education settings, including accessibility to a wide population and range of parents, pre-existing infrastructure that minimizes the need for additional resources to deliver the education, ability to accommodate a variety of teaching-learning methods, and flexibility in the timing and duration of education. We further examined the effectiveness of our parent-directed knowledge translation tools after provision to new parents in the hospital setting, specifically examining the effect of drawing parents' attention to the pain pamphlet with or without the video. We hypothesized that both the pain pamphlet alone and pain pamphlet plus pain video would increase parent utilization of pain interventions at future infant vaccinations compared with control, and that the pain pamphlet and pain video combined would be more effective than the pain pamphlet alone.

\section{Methods}

\section{Study population and setting}

We conducted a longitudinal, 3-group parallel, add-on randomized controlled trial (RCT). The study population was new mothers admitted to hospital who were rooming in with their newborn infants in the mother-and-baby unit at Mount Sinai Hospital, Toronto. Fathers could be included; however, this was not a requirement. We excluded mothers who were unable to communicate in English, those being treated for psychiatric conditions or those who were sharing a room with another mother who had already participated. Research assistants consulted with the charge nurse before approaching mothers about participation, as per institutional practices. We conducted the study between Oct. 26, 2013, and July 3, 2016.

Participants were randomly assigned to 1 of 3 intervention groups and 3 follow-up groups (i.e., 9 groups, $3 \times 3$ ). The intervention groups were a control group (participants received the general immunization pamphlet, available at http://publications.gc.ca/site/ eng/9.692169/publication.html, and the general immunization video, both of which were produced by the Public Health Agency of Canada); a group receiving the pain pamphlet (specific information about pain mitigation; hereafter called pain pamphlet) (Appendix 1 , available at www.cmaj.ca/lookup/suppl/doi:10.1503/cmaj. 180175/-/DC1) and general immunization video; and a group receiving the pain pamphlet and pain video (specific information about pain mitigation, available at www.youtube.com/watch?v=50qa1Fag5eQ\# action=share; hereafter called pain pamphlet and pain video). Participants randomly assigned to the pain education groups also received the general immunization pamphlet and video to prevent potential bias about perceived importance of immunization. The general immunization pamphlet contained some information about comforting infants, including advising mothers to relax, cuddle (including breastfeed) and distract during injections. The pain pamphlet included a link to the pain video. There were 3 follow-up groups comprising mothers whose infants were vaccinated at 2 months, those with infants vaccinated at 4 months and those with infants vaccinated at 6 months.

Participants were unaware of the study hypothesis. They were informed that the study was investigating whether information about vaccination should be added to the postnatal educational booklet given to all new parents. They were fully debriefed at the end of the study.

Research assistants accessed a central computer randomization service (randomize.net) from the nursing station to determine allocation after consent was obtained, then retrieved the appropriate materials from a locked research office and hand-delivered the allocated education to each participant. Research assistants provided a summary of the topics included and asked participants to review independently the materials (estimated time for review about 10-15 min). The research assistant turned the television on to a parent-accessible channel to access the video and noted whether the mother reviewed both the pamphlet and the video while they were in the room. Participants kept the pamphlets.

After discharge from hospital and 1 week before anticipated 2-, 4- and 6-month infant vaccinations, an automated reminder was sent to alert the participants to review the information. A followup telephone interview was conducted by a different research assistant, who was unaware of treatment allocation, after the randomized follow-up time to collect outcome data using a standardized questionnaire adapted from previous studies. ${ }^{14-16}$

\section{Primary and secondary outcomes}

The primary outcome was self-reported utilization (yes/no) of any of the 3 targeted interventions (breastfeeding, sucrose or topical anesthetics) at routine 2-, 4- or 6-month infant vaccinations. We previously showed that maternal recall of these particular interventions during infant vaccinations is accurate. ${ }^{14}$ Secondary outcomes included utilization of individual interventions, knowledge about interventions, perceptions about pain and pain management, and vaccination compliance. Knowledge was assessed using true/false questions and summing correct responses. Participants rated how sure they were of their answers, and only those that were correct and the participant was sure were correct were scored as correct. Perceptions about importance of pain, effect of pain management on maternal anxiety about vaccination, confidence in ability to mitigate pain and infant pain were assessed using ordinal and ratio scales. Unsuccessful utilization attempts (i.e., tried but failed) were also tracked. Compliance with vaccination was assessed by calculating infant age at vaccination. Reasons for not using interventions were recorded and categorized according to themes. 


\section{Sample size calculation}

A previous study involving 92 participants reported a 15\% higher utilization rate in 2 of the 3 targeted interventions for new mothers that read the pain pamphlet compared with those that did not. ${ }^{16}$ Using this difference, we calculated 215 participants per group (total 645) with a 2-tailed $\alpha$ of 0.017 (to account for 3 pairwise comparisons between groups) and power of $80 \%$ (NCSS and PASS statistical software, www.ncss.com). This number was tripled to account for secondary analyses of the primary outcome at 2-, 4and 6-month vaccinations and further increased by $33 \%$ to account for potential noncompliance and 33\% to account for follow-up losses (i.e., 1140 participants per group; total 3420).

\section{Statistical analysis}

We initially used a modified intention-to-treat analysis approach, with missing data excluded (i.e., complete case intention-to-treat analysis). Categorical and continuous data were compared using the $\chi^{2}$ test and analysis of variance (ANOVA), respectively. We repeated the analysis for attempts (i.e., successful and unsuccessful utilization combined). We conducted prespecified subgroup analyses by follow-up period (i.e., 2, 4 and $6 \mathrm{mo}$ ). We used logistic regression to examine predefined factors (group allocation, followup period, type of delivery, first-time mother, father present at education, maternal knowledge about pain interventions, perceived importance of treating vaccination pain, perceived importance of pain management on maternal anxiety about vaccination and confidence in ability to mitigate infant pain) and any variables that reflected imbalances at baseline on the primary outcome.

We then performed an adjusted analysis for the primary outcome whereby we examined the baseline characteristics of participants included in the analysis to determine if prognostic factors became unbalanced because of losses to follow-up and controlled for these baseline characteristics in the logistic regression analysis to determine the robustness of the results. ${ }^{19} \mathrm{We}$ used multiple imputation as an additional sensitivity analysis. Because the pattern for missing data was identified as arbitrary,

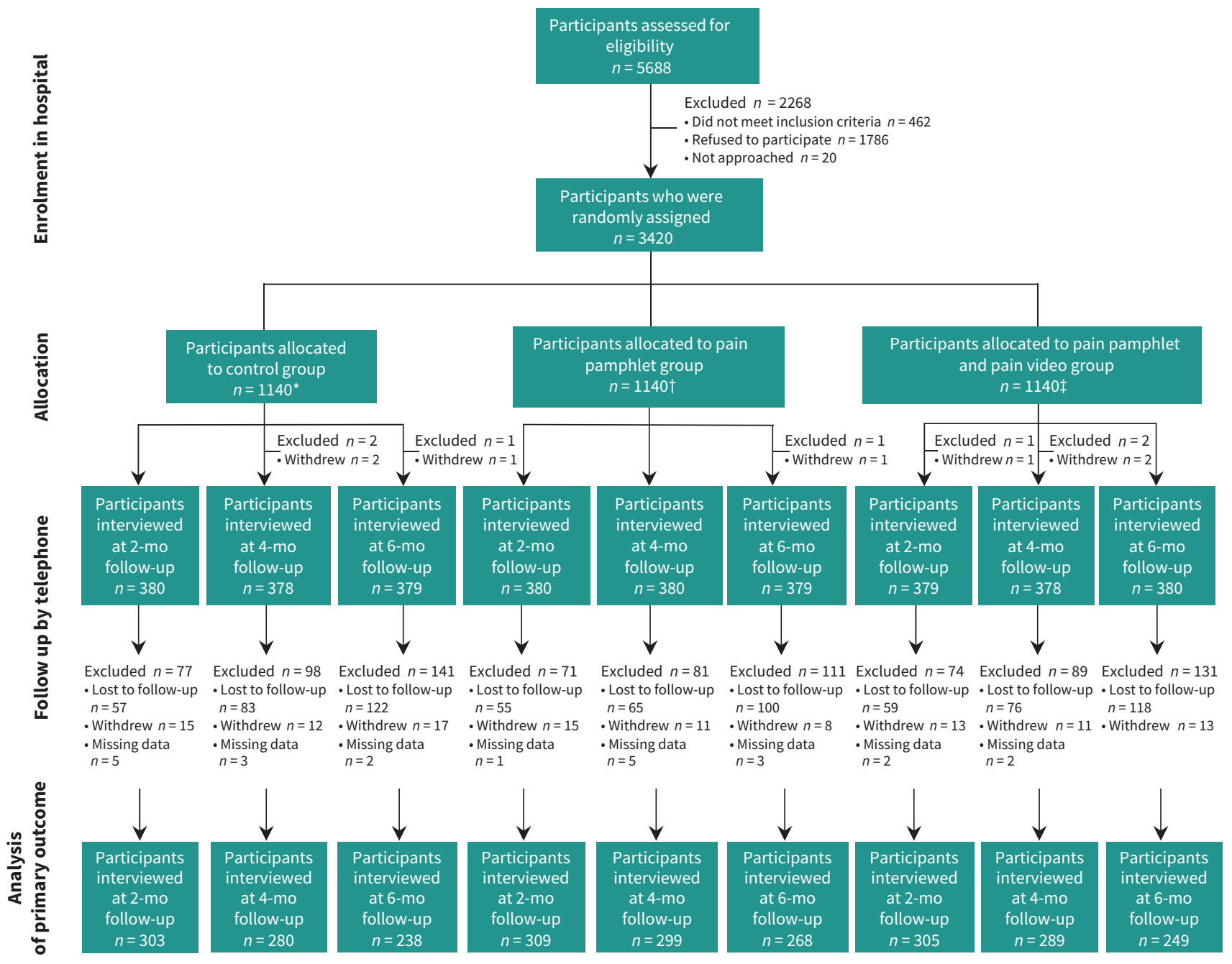

Figure 1: Flow diagram showing random allocation of participants, exclusions, follow-up and analysis groups. ${ }^{*}$ Control = participants received a pamphlet and video with general information about immunization. †Pain pamphlet = participants received a pamphlet about pain and a video with general information about immunization (and a pamphlet with general information about immunization). $\ddagger$ Pain pamphlet and pain video = participants received a pamphlet and video about pain (and a pamphlet and video with general information about immunization). 
we used the fully conditional specification method for imputing missing data for continuous and categorical data. ${ }^{20}$ Five complete data sets were generated that were analyzed and combined for the inference. All analyses were conducted using SAS (version 9.4). We set the level of significance at $p=0.05$.

\section{Ethics approval}

The study was approved by the Research Ethics Board at Mount Sinai Hospital (study centre), the University of Toronto and The Hospital for Sick Children.

\section{Results}

Of 5688 mothers assessed for eligibility, we included 3420 (60\%) (Figure 1). Fathers were present $64 \%$ of the time. One percent of participants did not review all the allocated materials at the time when they were given to them. Sixty-two participants $(1.8 \%)$ received incorrect education materials; we conducted analysis for these participants based on the group that they were randomly assigned to. We conducted follow-up interviews with 2549 (74.5\%) participants: 921 (36.1\%), 871 (34.2\%) and $757(29.7 \%)$ were interviewed after 2-, 4- and 6-month vaccinations, respectively. The primary outcome was available in 2540 participants (74.3\%). There was a difference in attrition rate among groups $(p=0.04)$. Post hoc pairwise comparisons showed higher attrition in the control group compared with the pain pamphlet group ( $p=0.01)$; there were no other differences. We found that maternal and birth characteristics did not differ among intervention groups (Table 1). Altogether, 1937 participants (76\%) identified 736 different treating physicians for infants.

The mean elapsed time between vaccination and follow-up was 39 (standard deviation [SD] 59) days in all groups ( $p=0.9)$. Table 2 provides the primary and secondary study outcomes by intervention group. The rate of use of any of the targeted pain interventions (breastfeeding, sucrose or topical anesthetics) was $53.2 \%, 61.4 \%$, and $63.0 \%$ for the control, pain pamphlet and combined pain pamphlet and pain video groups, respectively $(p<0.001)$. Both pain pamphlet groups had higher rates of utilization compared with the control group ( $p \leq 0.001$ for both analyses). When examined separately, we found that individual interventions were used more frequently in the pain education groups than in the control group. When we combined all attempts (successful and unsuccessful), the rates were 68\%, 75\% and $77 \%$ for control, pain pamphlet, and pain pamphlet and pain video groups, respectively $(p<0.001)$, with evidence of a significant difference between both pain education groups and the control group.

We found no significant differences between the pain education groups. Participants in both pain education groups scored higher on the knowledge test, had more positive perceptions about the importance of managing vaccination pain and perceived less pain in infants during vaccination.

Vaccination compliance (as assessed by mean infant age at vaccination) did not differ among groups. The most common reasons for not using interventions fit into 3 themes: knowledge, attitudes and intervention characteristics (Appendix 2, available at www.cmaj.ca/lookup/suppl/doi:10.1503/cmaj.180175/-/DC1).

When we compared intervention groups according to follow-up group (2-, 4- or 6-mo vaccinations), we observed differences in utilization of any of the 3 targeted interventions between both pain education groups compared with control at 2 and 4 months only (Table 3). Our logistic regression analysis showed a positive effect on utilization according to intervention group (both pain education groups v. control group), follow-up group ( 2 and 4 mo v. 6 mo), birth by vaginal delivery, multiparous participant, knowledge about pain interventions, perception that pain mitigation is important, and perception that mitigating pain reduces anxiety about vaccination (Table 4).

Table 1: Characteristics of participants, by intervention group

No. (\%) of participants

\section{Characteristic}

Maternal age, mean \pm SD; $y r$

Education level, postsecondary

Marital status, married/common law

Primiparous

Mode of delivery, vaginal

Singleton birth

Gestational age, mean \pm SD; $d$

Infant age at intervention, mean \pm SD; yr
Control group

$n=1137$

$33.5 \pm 4.6$

1055 (93.9)

$n=1123$

1086 (95.3)

$n=1140$

669 (58.8)

729 (64.1)

1117 (98.2)

$275 \pm 9.1$

$1.0 \pm 0.7$
Pain pamphlet group

$n=1139$

$33.6 \pm 4.6$

1062 (93.9)

$n=1131$

1084 (95.1)

$n=1140$

$666(58.5)$

$722(63.4)$

1124 (98.7)

$275 \pm 8.6$

$1.0 \pm 0.6$
Pain pamphlet and pain video group $n=1137$

$33.6 \pm 4.8$

1056 (93.9)

$n=1125$

$1083(95.0)$

$n=1140$

$646(56.8)$

$737(64.8)$

$1122(98.7)$

$275 \pm 9.1$

$1.0 \pm 0.7$

$n=1136$ 
When we compared the characteristics of the subsample of participants that were followed-up among groups, we found no evidence of a difference (Appendix 3, available at www.cmaj.ca/ lookup/suppl/doi:10.1503/cmaj.180175/-/DC1). The adjusted logistic regression analysis, which controlled for these factors, showed similar results to the initial analysis (Appendix 4, available at www.cmaj.ca/lookup/suppl/doi:10.1503/cmaj.180175/-/ $D C 1)$. The results for the multiple imputation analysis are shown in Appendix 5, available at www.cmaj.ca/lookup/suppl/ doi:10.1503/cmaj.180175/-/DC1; results were similar.

\section{Interpretation}

New parents who were given education about pain mitigation during infant vaccination using a pain pamphlet or pain pamphlet and companion video, in addition to receiving general information about immunization, used breastfeeding, sucrose or topical anesthetics more frequently at future infant vaccinations compared with those that received general immunization information only. The absolute increase in utilization of 1 or more of these interventions was $8 \%$ for the pain pamphlet and $10 \%$ for

\section{Table 2: Utilization of pain interventions and secondary outcomes, by intervention group}

No. $(\%)$ of participants*

\section{Characteristic}

Utilization of pain interventions at infant vaccinations

Used any intervention $\ddagger$

Used breastfeeding

Used sucrose solution

Used topical anesthetic

Attitudes and beliefs about pain and pain management

Participant stated that vaccination pain is important to manage

Participant believed what they did helped

Participant was satisfied with pain management

Score for participant confidence in ability to manage vaccination pain $(0-10)$, mean $\pm S D$

Score for participant-reported infant pain at vaccination (0-10), mean \pm SD

\section{Knowledge about pain interventions}

Participant score on knowledge test (0-3), mean \pm SD 9

\section{Compliance with vaccinations}

Infant age at vaccination, mean \pm SD; $d$

Infant age at 2 mo vaccination, mean \pm SD; $d$

Infant age at 4 mo vaccination, mean \pm SD; $d$

Infant age at 6 mo vaccination, mean $\pm S D ; d$

\section{Control group}

$\begin{array}{cc}437(53.2)^{\mathrm{a}, \mathrm{b}} & 538(61.4)^{\mathrm{a}} \\ n=821 & n=876 \\ 422(51.2)^{\mathrm{a}, \mathrm{b}} & 502(57.3)^{\mathrm{a}} \\ n=824 & n=876 \\ 25(3.0)^{\mathrm{a}, \mathrm{b}} & 71(8.1)^{\mathrm{a}} \\ n=823 & n=878 \\ 15(1.8)^{\mathrm{b}} & 26(3.0)^{\mathrm{c}} \\ n=821 & n=876\end{array}$

$$
\begin{gathered}
672(81.5)^{\mathrm{a}, \mathrm{b}} \\
n=825 \\
700(85.2)^{\mathrm{b}} \\
n=822 \\
715(87.2)^{\mathrm{a}, \mathrm{b}} \\
n=820 \\
7.0 \pm 2.2^{\mathrm{a}, \mathrm{b}} \\
n=820 \\
6.0 \pm 2.1^{\mathrm{a}, \mathrm{b}} \\
n=524
\end{gathered}
$$

$$
\begin{gathered}
1.1 \pm 0.9^{\mathrm{a}, \mathrm{b}} \\
n=815
\end{gathered}
$$

$121 \pm 51$

$$
n=826
$$$$
67 \pm 14
$$$$
n=305
$$$$
124 \pm 14
$$$$
n=282
$$$$
187 \pm 20
$$$$
n=239
$$

Pain pamphlet

group

\section{Pain pamphlet and pain video group} $p$ valuet
Omnibus test

Note: $\mathrm{SD}=$ standard deviation

*Unless specified otherwise.

tOmnibus test ( $\chi^{2}$ or mixed methods) $p$ value for comparisons among the 3 treatment groups. Post hoc pairwise comparisons with $p$ values $<0.05$ are denoted by the following

symbols: $\mathrm{a}=$ control group $\mathrm{v}$. pain pamphlet group, $\mathrm{b}=$ control group $\mathrm{v}$. pain pamphlet and pain video group and $\mathrm{c}=$ pain pamphlet group $\mathrm{v}$. pain pamphlet and pain video group. flnterventions included breastfeeding, sucrose solution and topical anesthetic.

IThe following script and questions were read to all mothers and each correct answer was given a score of 1 (total range, 0-3). "Can you tell me what you know now about whether these things actually work to lessen a baby's pain from needles (i.e., as the needle is going in)? 1. Giving a baby sugar water to drink reduces pain. 2 . Breastfeeding a baby reduces pain. 3. Using numbing medicines such as topical anesthetics reduces pain. 
the pain pamphlet and pain video, with corresponding numbers needed to treat of 13 and 10 , respectively. We found no evidence of a difference when the pain pamphlet was used alone compared with when it was combined with the pain video. In addition, knowledge about mitigation of pain during vaccinations was higher, there were more positive perceptions about pain and pain management, and less perceived infant pain for these two groups. There was no evidence of a difference in compliance with vaccination among groups.

The observed effect is lower than the estimated absolute benefit of $15 \%$; however, it is consistent with improvements in care achieved in studies of knowledge translation interventions involving distribution of educational materials (i.e., 8.1\%). ${ }^{21}$ Our findings add to work aimed at increasing uptake of a national clinical practice guideline for management of vaccination pain. ${ }^{13-}$ ${ }^{16}$ In 2 studies that delivered the same tools using a more active educational approach, either featuring the pamphlet and video in prenatal classroom teaching or in an outpatient pediatric clinic (including looping the video on the clinic's reception-area television and mounting posters on the wall), higher uptake rates were reported for pain interventions (absolute increase $17 \%$ and $26 \%$, respectively). ${ }^{14,15}$ Conversely, in a hospital-based study, the effect was limited when the pain pamphlet was passively disseminated in the hospital postnatal discharge information package. ${ }^{16}$ Effect was associated with the parent reading the pamphlet; most did not. Our study used the hospital setting but actively presented the pain education to new parents with verbal instructions to review it. This small difference in the method of education delivery resulted in a significant positive effect, albeit smaller than anticipated. The contribution of timing of the education on education effect is not known. In addition, although the lack of a difference between the 2 pain education groups suggests that the video was not needed, the design did not allow for a determination of whether the video alone would achieve the same effect. Level of health literacy may affect the relative benefits of viewing a video in addition to and compared with written education, ${ }^{22}$ and participants in our study were highly educated.
The negative findings with respect to effect on vaccination compliance (as determined by infant age) could be due to use of reminders that promoted compliance in all groups, ${ }^{23}$ refusal of parents who were vaccine hesitant to participate in a study about vaccination and attrition of parents who were noncompliant. We did not know the distribution of noncompliance among participants lost to follow-up and could have included some parents in the intervention groups who were scared about vaccination because of infant pain.

The high rate of utilization of breastfeeding that we observed warrants discussion. ${ }^{8,14-16}$ The hospital involved in the study routinely employs breastfeeding during the screening test for newborns, and mothers may have transferred this knowledge to infant vaccinations. Community uptake of this pain intervention may have increased because of adoption of guideline recommendations. ${ }^{4,5}$ Information about breastfeeding was included in the control pamphlet, and although it was not prominently featured, this may have been sufficient to result in education about breastfeeding in all groups. There may be differences in how we measured breastfeeding. We included breastfeeding undertaken before, during or after needle injection. Although it is optimal to breastfeed during actual injection of the vaccine, there is documented benefit for breastfeeding undertaken before injection as well. ${ }^{5}$ Separately, there is evidence for effectiveness of physical interventions involving holding and sucking after injection. ${ }^{5}$

The lower scores for infant pain scores that were reported by participants in active groups provides evidence for real-world effectiveness of targeted pain interventions that are provided by parents. We also note that rotavirus vaccine administered orally, which is given in conjunction with injectable vaccines at 2 and 4 months, contains sucrose. A randomized trial published in 2015 reported that infant distress during vaccine injections did not differ between infants given rotavirus vaccine and sucrose solution before injectable vaccines. ${ }^{24}$ Therefore, administration of rotavirus vaccine before injectable vaccines in lieu of sucrose may confer the added benefit of pain relief.

Table 3: Utilization of pain interventions, by intervention group and follow-up group

No. $(\%)$ of participants
Characteristic

Infant vaccination at $\mathbf{2}$ mo

Used any interventiont

\section{Infant vaccination at $\mathbf{4}$ mo}

Used any interventiont

Infant vaccination at 6 mo

Used any interventiont
Control group

$$
\begin{gathered}
169(55.8)^{\mathrm{a}, \mathrm{b}} \\
n=303
\end{gathered}
$$

$150(53.6)^{\mathrm{a}, \mathrm{b}}$

$$
n=280
$$

$118(49.6)$

$n=238$
Pain pamphlet

Pain pamphlet group
Omnibus test, $p$ value* $198(64.9)^{\mathrm{b}}$ $n=305$

and pain video group

$99(64.4)^{\circ}$

$n=309$

$189(63.2)^{\mathrm{a}}$

$n=299$ $190(65.7)^{\mathrm{b}}$
$n=289$

$143(57.4)$

$n=249$
0.03

$\begin{array}{cc}150(56.0) & 143(57.4) \\ n=268 & n=249\end{array}$

0.2
${ }^{*}$ Omnibus test $\left(\chi^{2}\right) p$ value for comparisons among the 3 treatment groups. Post hoc pairwise comparisons with $p$ values $<0.05$ are denoted by the following symbols: a $=$ control group v. pain pamphlet, $\mathrm{b}=$ control group $\mathrm{v}$. pain pamphlet and pain video and $\mathrm{c}=$ pain pamphlet $\mathrm{v}$. pain pamphlet and pain video.

tInterventions included breastfeeding, sucrose solution and topical anesthetic. 
Table 4: Logistic regression analysis of factors affecting utilization of pain interventions

\section{Variable}

OR $(95 \% \mathrm{CI})$

\section{Grouping variable}

Group, 2 (pain pamphlet) v. 1 (control)

Group, 3 (pain pamphlet and pain video) v. 1 (control)

Group, 3 (pain pamphlet and pain video) v. 2 (pain pamphlet)

\section{Other variables}

Attitude, mother confident, 0 (no) v. 1 (yes)

$0.92(0.76-0.98)$

Attitude, mother less anxious, 0 (no) v. 1 (yes)

$0.78(0.65-0.92)$

Attitude, pain is important, 0 (no) v. 1 (yes)

$0.76(0.60-0.96)$

Knowledge, continuous (0-3)

$1.32(1.21-1.44)$

Follow-up date, 2 ( $4 \mathrm{mo}$ ) v. 1 ( $2 \mathrm{mo}$ )

$0.93(0.76-1.14)$

Follow-up date, 3 (6 mo) v. 1 (2 mo)

$0.73(0.59-0.90)$

Follow-up date, 3 (6 mo) v. 2 (4 mo)

$0.78(0.63-0.96)$

Mode of delivery, 0 ( cesarian section) v. 1

(vaginal)

Multipara, 0 (no) v. 1 (yes)

$0.65(0.55-0.78)$

Father present at education, 0 (no) v. 1 (yes)

$0.69(0.58-0.82)$

$0.95(0.79-1.13)$

Note: $\mathrm{Cl}=$ confidence interval, $\mathrm{OR}=$ odds ratio

Our study has numerous strengths, including random sequence generation, allocation concealment, blinding of participants and outcome assessors. ${ }^{25}$ Contamination of controls was minimized by having participants review materials in their own hospital room, independent of others. If participants shared a room, only 1 participated. General information about vaccination was given to all participants to prevent bias created about the perceived importance of immunization, which could affect compliance. Conducting interviews at different follow-up times enabled evaluation of intervention sustainability, while preventing contamination and reporting bias from repeat interviews with the same participants. External validity was enhanced by using pragmatic design features. ${ }^{26}$ These included minimal exclusion criteria, feasible education delivery, vaccination of infants in many practice settings and measurement of clinically relevant outcomes.

\section{Limitations}

There are limitations to our study. First, the primary outcome was obtained using self-reporting by participants. However, we previously showed that mothers are reliable when reporting on the targeted interventions. ${ }^{14}$ Second, attrition was greater than $20 \%$, which may have introduced bias. However, our adjusted analysis of the primary outcome, including prognostic factors and multiple imputation showed similar results. There was a higher rate of attrition in the control group compared with the pamphlet group; there are no clear implications of this isolated finding. We do not have an explanation for the differential attrition. Third, the study was overpowered and could detect small differences between groups. Fourth, the included sample represented $60 \%$ of eligible participants. Mean age, parity and education of the study population may differ from the broader public, affecting generalizability. Fifth, we did not assess infant pain by using behavioural analysis of videotapes, which is the gold standard approach employed in pain studies. However, the primary purpose of the study was to determine utilization of interventions for pain mitigation and not their effectiveness, which had already been established in previous studies, ${ }^{5}$ and videotaping infants was impractical given the large number of practice settings. Parental assessments correlate with videotape analyses, provide real-time clinical validation of the effectiveness of the interventions and were considered acceptable. ${ }^{5}$ Sixth, the optimal timing for postnatal education in the hospital setting is not known, and further research on this topic has been suggested. ${ }^{18}$ Finally, inclusion of information about breastfeeding in the pamphlet provided to the control group may have led to information contamination in the control group. However, this would have resulted in a more conservative estimate of intervention effects.

\section{Conclusion}

In summary, we found that when new parents learned about vaccine pain mitigation techniques before discharge from the hospital using feasible knowledge translation tools (pamphlet and video) and delivery methods that mimic current postnatal educational practices, there is a small increase in uptake of pain interventions at future infant vaccinations. Knowledge, skills and confidence in their abilities to mitigate infant pain also increase. These results should be interpreted in light of the feasibility of the intervention. The tools used in this study are portable and can be made available in a variety of education formats and platforms, and educatorled administration formats. This education can be added to existing education given to new parents at minimal cost. More research is required to address barriers to intervention use, particularly with respect to addressing learner knowledge and attitudes about pain management. Involving health care providers in education and provision of pain interventions and making information available in different formats and settings may bolster implementation success. Further evaluation of the influence of pain mitigation on vaccination compliance and infant attachment, critical aspects of healthy child development, are also warranted.

\section{References}

1. Taddio A, Chambers C, Halperin S, et al. Inadequate pain management during childhood immunization: the nerve of it. Clin Ther 2009;31(Suppl 2):S152-7.

2. World Health Organization. Reducing pain at the time of vaccination: WHO position paper - September 2015. Wkly Epidemiol Rec 2015;90:505-10.

3. MacDonald NE; SAGE Working Group on Vaccine Hesitancy. Vaccine hesitancy: Definition, scope and determinants. Vaccine 2015;33:4161-4.

4. Taddio A, Appleton M, Bortolussi B, et al. Reducing the pain of childhood vaccination: an evidence-based clinical practice guideline. CMAJ 2010;182:E843-55.

5. Taddio A, McMurtry CM, Shah V, et al.; HELPinKids\&Adults. Reducing pain during vaccine injections: clinical practice guideline. CMAJ 2015;187:975-82.

6. Taddio A, Manley J, Potash L, et al. Routine immunization practices: use of topical anesthetics and oral analgesics. Pediatrics 2007;120:e637-43.

7. Taddio A, Ipp M, Thivakaran S, et al. Survey of the prevalence of immunization non-compliance due to needle fears in children and adults. Vaccine 2012;30:4807-12.

8. Harrison D, Sonja Elia S, Royle J, et al. Pain management strategies used during early childhood immunisation in Victoria. J Paediatr Child Health 2013;49:313-8 
9. Betsch C, Bodeker B, Schmid P, et al. How baby's first shot determines the development of maternal attitudes towards vaccination. Vaccine 2018; 36:3018-26.

10. Letourneau N, Tryphonopoulos P, Giesbrecht G, et al. Narrative and metaanalytic review of interventions aiming to improve maternal-child attachment security. Infant Ment Health J 2015;36:366-87.

11. Simpson TE, Condon E, Price RM, et al. Demystifying infant mental health: what the primary care provider needs to know. J Pediatr Health Care 2016;30:38-48.

12. Taddio A, Shah V, Leung E, et al. Knowledge translation of the HELPinKIDS clinical practice guideline for managing childhood vaccination pain: usability and knowledge uptake of educational materials directed to new parents. BMC Pediatr 2013;13:23.

13. Taddio A, Ipp M, Vyas $C$, et al. Teaching parents to manage pain during infant immunizations: laying the foundation for better pain management practices. Clin J Pain 2014;30:987-94.

14. Taddio A, Smart S, Sheedy M, et al. Impact of prenatal education on maternal utilization of analgesic interventions at future infant vaccinations: a cluster randomized trial. Pain 2014;155:1288-92.

15. Taddio A, Parikh C, Yoon E, et al. Impact of parent-directed education on parental use of pain treatments during routine infant vaccinations: a cluster randomized trial. Pain 2015;156:185-91.

16. Taddio A, MacDonald NE, Smart S, et al. Impact of a parent-directed pamphlet about pain management during infant vaccinations on maternal knowledge and behavior. Neonatal Netw 2014;33:74-82.
17. Grol R, Grimshaw J. From best evidence to best practice: effective implementation of change in patients' care. Lancet 2003;362:1225-30.

18. Bryanton J, Beck CT, Montelpare W. Postnatal parental education for optimizing infant general health and parent-infant relationships. Cochrane Database Syst Rev 2013; (11):CD004068.

19. Groenwold RHH, Moons KGM, Vandenbroucke JP. Randomized trials with missing outcome data: how to analyze and what to report. CMAJ 2014;186:1153-7.

20. The MI procedure. In: SAS/STAT ${ }^{\circledR} 13.2$ user's guide. Cary (NC): SAS; 2014. Available: http://support.sas.com/documentation/cdl/en/statug/67523/HTML/ default/viewer.htm\#statug_mi_details05.htm (accessed 2018 July 25).

21. Grimshaw J, Eccles M, Thomas R, et al. Evidence and its limitations of the effectiveness of guideline dissemination and implementation strategies 1966 1998. J Gen Intern Med 2006;21:S14-20.

22. Lambert V, Keogh D. Health literacy and its importance for effective communication. Part 1. Nurs Child Young People 2014;26:31-8.

23. Harvey $\mathrm{H}$, Reissland N, Mason J. Parental reminder, recall and educational interventions to improve early childhood immunisation uptake: a systematic review and meta-analysis. Vaccine 2015;33:2862-80.

24. Taddio A, Flanders D, Weinberg E, et al. A randomized trial of rotavirus vaccine versus sucrose solution for vaccine injection pain. Vaccine 2015;33:2939-43.

25. Hartling L, Hamm M, Klassen T, et al. Standard 2: Containing risk of bias. Pediatrics 2012;129:S124-31.

26. Thorpe KE, Zwarenstein M, Oxman AD, et al. A pragmatic-explanatory continuum indicator summary (PRECIS): a tool to help trial designers. CMAJ 2009;180:E47-57.
Competing interests: Anna Taddio has received a research grant from Pfizer (investigator initiated) and supplies for a separate study from Natus Medical and Ferndale Pharma Group. Lucie Bucci has received research grants from Pfizer Canada, Merck Canada, Sanofi Pasteur, GlaxoSmithKline and Seqirus Canada. No other competing interests were declared.

This article has been peer reviewed.

Affiliations: Leslie Dan Faculty of Pharmacy (Taddio, Wong), University of Toronto; Department of Pediatrics (Shah), Mt. Sinai Hospital, Toronto, Ont.; Immunize Canada (Bucci), Canadian Public Health Association, Ottawa, Ont.; Department of Paediatrics, Faculty of Medicine (MacDonald), Dalhousie
University, Halifax, NS; Biostatistics and Data Analysis Unit (Stephens), The Hospital for Sick Children, Toronto, Ont.

Contributors: Anna Taddio, Vibhuti Shah, Lucie Bucci, Noni MacDonald and Derek Stephens conceptualized and designed the study. Anna Taddio, Vibhuti Shah and Horace Wong assisted in the acquisition and interpretation of the data. Anna Taddio and Derek Stephens conducted the analysis. Anna Taddio and Vibhuti Shah provided administration of and supervision for the study. All of the authors reviewed the manuscript critically for important intellectual content and revised it accordingly, gave final approval for the version to be published and agreed to be accountable for all aspects of the work.
Funding: Funding for the study was acquired by Anna Taddio from the Canadian Institutes of Health Research (MCT 126007). The Canadian Institutes for Health Research had no role in design and conduct of the study; collection, management, analysis, and interpretation of the data; and preparation, review or approval of the manuscript.

Data sharing: Data from this study are not available.

Acknowledgements: The authors thank Eshetu Atenafu, Linda Dodd and Christy Woolcott for their statistical advice.

Accepted: Sept. 6, 2018

Correspondence to: Anna Taddio, anna. taddio@utoronto.ca 